\title{
Design of power system stabilizer for damping power system oscillations
}

\author{
Ahmed Mohammed.Ishag ${ }^{1}$, Eisa B. M. Tayeb ${ }^{2}$ \\ ${ }^{1,2}$ (College of Engineering/Sudan Universities of Science and Technology, Sudan)
}

\begin{abstract}
The problem of the poorly damped low-frequency (electro-mechanical) oscillations of power systems has been a matter of concern to power engineers for a long time, because they limit power transfers in transmission lines and induce stress in the mechanical shaft of machines. Due to small disturbances, power systems experience these poorly damped low-frequency oscillations. The dynamic stability of power systems are also affected by these low frequency oscillations. With proper design of Power System Stabilizer (PSS), these oscillations can be well damped and hence the system stability is enhanced. The basic functions of the PSS is to add a stabilizing signal that compensates the oscillations of the voltage error of the excitation system during the dynamic/transient state, and to provide a damping component when it's on phase with rotor speed deviation of machine. Studies have shown that PSS are designed to provide additional damping torque, for different operation point normal load, heavy load and leading to improve power system dynamic stability.
\end{abstract}

Keywords: dynamic stability, low frequency oscillation, power system stabilizer, small signal stability.

\section{Introduction}

In recent years, as interconnected power systems are becoming more and more complex, the subsistence of poorly damped low-frequency inter-area oscillations have strained its stability constraints, and thereby, limited its power transfer capacity. Therefore, adequate damping of inter-area modes is paramount in order to secure system operation, ensure system reliability and increase power generally; stability of power system is defined as "the ability of an electric power system, for a given initial operating condition, to regain a state of operating equilibrium after being subjected to a physical disturbance". Oscillatory stability is a subcategory of Small Signal Stability (SSS) which is defined as the ability of power system to maintain synchronous operation under small disturbances. The oscillations usually are concerned with frequencies between 0.2 to 3 hertz with insufficient damping. There are three types of oscillations that have been observed in the power system which are inter-unit oscillations, involves typically two or more synchronous machines at a power plant or nearby power plants swing against each other, with the frequency ranging between $1.5 \mathrm{~Hz}$ to 3 $\mathrm{Hz}$, local mode oscillations generally involve one or more synchronous machines at a power stations winging together against a comparatively large power system or load center and the frequency of oscillation is in the range of $0.7 \mathrm{~Hz}$ to $2 \mathrm{~Hz}$, finally, inter-area oscillations which involve combinations of many machines on one part of the power system swinging against machines on another part of the power system and the frequency range is normally less than $0.5 \mathrm{~Hz}$ [1].

Controlling these kinds of oscillations is relatively a simple task that could be solved by applying Power System Stabilizer (PSS) [2]. Different methods for analyzing the problem have been proposed by researchers in this area which could be found In1969 [3] De Mello and Concordia discussed the phenomena of Stability of synchronous machines under small perturbations by examining the case of a SMIB through external reactance. The analysis developed insights into effects of thyristor-type excitation systems and established understanding of the stabilizing requirements for such systems. These stabilizing requirements included the voltage regulator gain parameters as well as the transfer function characteristics for a machine speed derived signal along with the voltage regulator reference for providing damping of machine oscillations. The study had explored a variety of machine loadings, machine inertias, and system external impedances with a determination of the oscillation and damping characteristics of voltage or speed following a small disturbance in mechanical torque. An attempt had been made to develop some unifying concepts that explain the stability phenomena of concern, and to predict desirable phase and magnitude characteristics of stabilizing functions.

In 1989 Kundur et al. [4] provided the analytical work and systematic method to determine PSS parameters for large power generation in a practical power system. The basic PSS design idea based on the stabilizer proposed in [3]. However, the phase characteristics were obtained using a multi-machine eigenvalue program instead of a single machine model. This work emphasized enhancement of overall system stability, and the authors considered simultaneous damping of inter-area and local modes and discussed the performance of the PSS under different system conditions. In addition to small signal stability performance, the authors also tested the transient stability performance of the PSS and the performance during system is landing. The authors also demonstrated the importance of appropriate choice of washout time constant, stabilizer output limits and 
other excitation system control parameters. The authors claimed that the frequency response method used to compensate the lag between the excitation input and the electrical torque was fairly robust.

Chow and Sanchez-Gasc in [5], proposed four pole-placement techniques for the design of power system stabilizers, with the emphasis on frequency response characteristics of the controller. For controllers to exhibit desirable frequency response characteristics, a simple procedure was proposed to obtain controllers suitable for multiple operating conditions.

\section{Mathematical model}

This section presents the small-signal model for a single machine connected to a large system through a transmission line (infinite bus) to analyze the local mode of oscillations in the range of frequency $1-3 \mathrm{~Hz}$. A schematic representation of this system is shown in Fig. 1 [6]

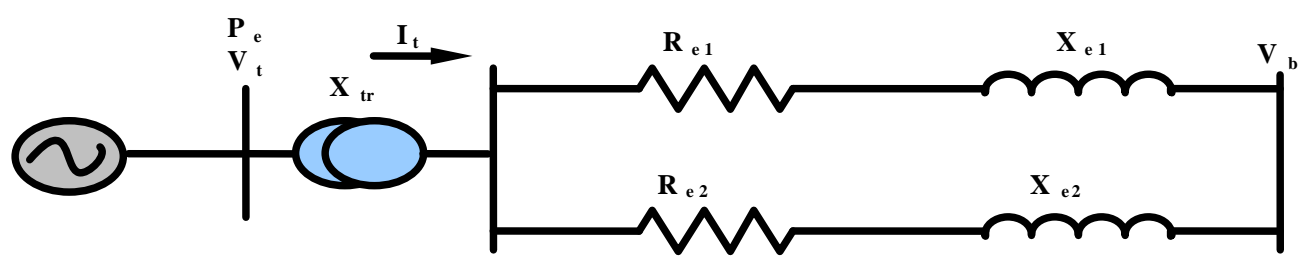

Figure 1 schematic diagram of single machine connected infinite bus

2.1 The Nonlinear equation of SMIB equipped with PSS:

$$
\begin{aligned}
& \frac{d \delta}{d t}=\omega b(\omega-\omega s) \\
& \frac{d \omega}{d t}=\frac{1}{M}[T m-T e-D(\omega-\omega s)] \\
& \frac{d E_{q}}{d t}=\frac{1}{T_{d 0}}\left[-E_{q}-\left(x_{d}-\hat{x}_{d}\right) i_{d}+E_{f d}\right] \\
& \frac{d E_{f d}}{d t}=\frac{1}{T_{A}}\left[-E_{f d}+K_{A}\left(V_{\text {ref }}-V_{t}+V_{s}\right)\right]
\end{aligned}
$$

\section{Power System Stabilizer Structure}

The basic objective of power system stabilizer is to modulate the generator's excitation in order to produce an electrical torque at the generator proportional to the rotor speed $[6,7]$. In order to achieve that, the PSS uses a simple lead-lag compensator circuit to adjust the input signal and correct the phase lag between the exciter input and the electrical torque. The PSS can use various inputs, such as the speed deviation of the generator shaft, the change in electrical power or accelerating power, or even the terminal bus frequency. However in many instances the preferred signal input to the PSS is the speed deviation. Fig. 2 below illustrates the block diagram of a typical PSS. The PSS structure generally consists of a washout, lead-lag networks, a gain and a limiter stages. . Each stage performs a specific function.

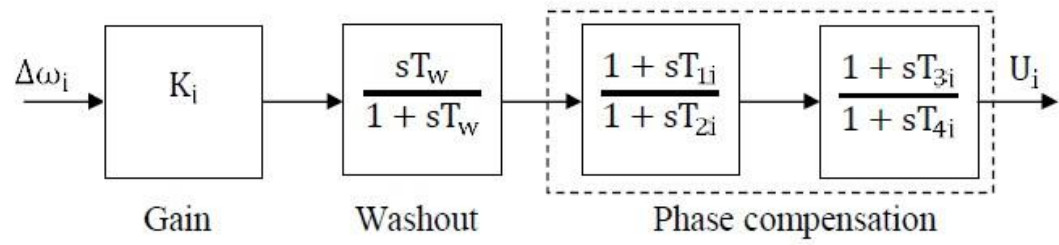

Figure 2 illustrates the block diagram of a typical PSS

$$
\begin{aligned}
& \frac{d V_{1}}{d t}=K_{P S S} \frac{d \Delta \omega}{d t}-\frac{1}{T_{W}} V_{1} \\
& \frac{d V_{2}}{d t}=\frac{T_{1}}{T_{2}} \frac{d V_{1}}{d t}+\frac{1}{T_{2}} V_{1}-\frac{1}{T_{2}} V_{2}
\end{aligned}
$$




$$
\frac{d V_{P S S}}{d t}=\frac{T_{3}}{T_{4}} \frac{d V_{2}}{d t}+\frac{1}{T_{4}} V_{2}-\frac{1}{T_{4}} V_{P S S}
$$

3.1 Linearization the system equipped with Static Excitation System:

$$
\begin{aligned}
& \Delta \dot{\delta}=\omega_{s} \Delta \omega \\
& \Delta \dot{\omega}=-\frac{K_{2}}{2 H} \Delta \dot{E}_{q}-\frac{K_{1}}{2 H} \Delta \delta-\frac{D \omega_{\Omega}}{2 H} \Delta \omega+\frac{1}{2 H} \Delta T_{M} \\
& \Delta \dot{E}_{q}=\frac{-1}{K_{a} \stackrel{\leftrightarrow}{T}_{d 0}} \Delta \dot{E}_{q}-\frac{K_{4}}{\dot{T}_{d 0}} \Delta \delta+\frac{1}{T_{d 0}} \Delta E_{f d} \\
& \Delta \dot{E}_{f d}=-\frac{K_{A} K_{6}}{T_{A}} \Delta \dot{E}_{q}-\frac{K_{A} K_{5}}{T_{A}} \Delta \delta-\frac{1}{T_{A}} \Delta E_{f d}+\frac{K_{A}}{T_{A}} \Delta V_{r e f} \\
& \text { Where, } \\
& K_{1}=\frac{-1}{\Delta}\left[I_{q} * E_{b *}\left(\grave{X}_{d}-X_{q}\right)\left\{\left(X_{q}+X_{e}\right) * \sin \delta-R_{e} * \cos \delta\right\}+E_{b} *\right. \\
& \left.\left\{\left(\grave{X}_{d}-X_{q}\right) * I_{d}-\grave{E}_{q}\right\}\left\{\left(\grave{X}_{d}+X_{e}\right) * \cos \delta+R_{e} * \sin \delta\right\}\right] \\
& K_{2}=\frac{1}{\Delta}\left[I_{q} * \Delta-I_{q} *\left(\grave{X}_{d}-X_{q}\right) *\left(X_{q}+X_{e}\right)-R_{e} *\left(\grave{X}_{d}-X_{q}\right) * \grave{E}_{q}\right] \\
& K_{3}=\frac{1}{1+\frac{\left(X_{d}-\tilde{X}_{d}\right) \in\left(X_{Q}+X_{Q}\right)}{\Delta}} \\
& K_{4}=\frac{E_{b *}\left(X_{d}-\grave{X}_{d}\right)}{\Delta}\left[\left(X_{q}+X_{e}\right) * \sin \delta-R_{e} * \cos \delta\right] \\
& K_{5}=\frac{1}{\Delta}\left\{\frac{V_{d}}{V t} * X_{q}\left[R_{e} * E_{b *} \sin \delta+E_{b *} \cos \delta *\left(\grave{X}_{d}+X_{e}\right)\right]+\frac{V_{q}}{V t}\left[\grave{X}_{d} *\right.\right. \\
& \left.\left.\left(R_{e} * E_{b *} \cos \delta-E_{b *}\left(X_{q}+X_{e}\right) * \sin \delta\right)\right]\right\} \\
& K_{6}=\frac{1}{\Delta}\left\{\frac{V_{d}}{V t} * X_{q} * R_{e}-\frac{V_{q}}{V t} * \grave{X}_{d} *\left(X_{q}+X_{e}\right)\right\}+\frac{V_{q}}{V t}
\end{aligned}
$$

\subsection{Modeling the system in Heffron Philips form:}

The Heffron-Phillips model of a synchronous machine has successfully been used for investigating the low frequency oscillations and designing power system stabilizers, fig. 3 show Heffron Philips structure.

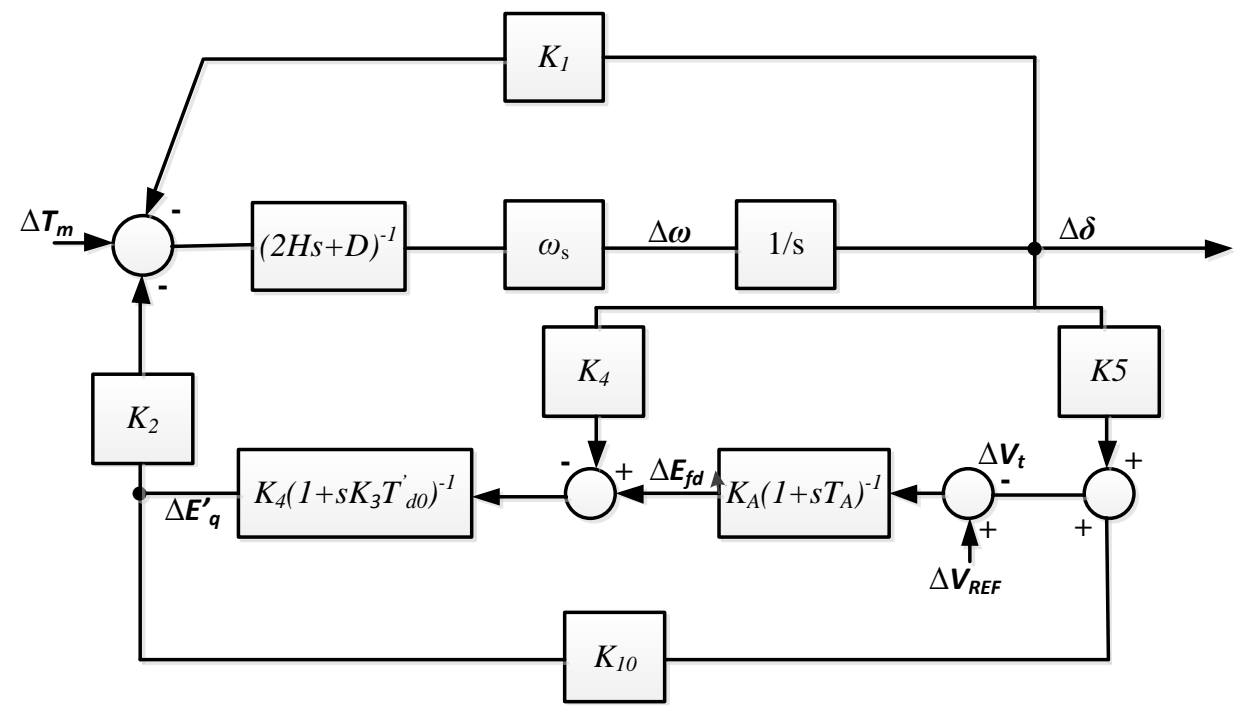

Figure 3: Heffron Philips model structure 


\subsection{Modeling the system in a state space form without PSS}

$$
[\Delta \dot{\mathrm{X}}]=[A][\Delta \mathrm{X}]+[B][\Delta \mathrm{U}]
$$

Where;

$$
\begin{aligned}
& {[\Delta X]=\left[\begin{array}{llll}
\Delta E_{q} & \Delta \delta & \Delta \omega & \Delta E_{f d}
\end{array}\right]^{T}} \\
& {[\Delta U]=\left[\Delta V_{R E F}\right]} \\
& {[A]=\left[\begin{array}{cccc}
\frac{-1}{K_{2} \hat{T}_{d 0}} & \frac{-K_{4}}{\grave{T}_{\text {do }}} & 0 & -\frac{1}{T_{d o}} \\
0 & 0 & \omega_{\text {base }} & 0 \\
\frac{-K_{2}}{M} & \frac{-K_{1}}{M} & \frac{-\omega_{\text {base }} K_{D}}{M} & 0 \\
-\frac{K_{6} K_{A}}{T_{A}} & -\frac{K_{5} K_{A}}{T_{A}} & 0 & -\frac{1}{T_{A}}
\end{array}\right] ;} \\
& {[B]=\left[\begin{array}{lllll}
0 & 0 & 0 & \frac{K_{A}}{T_{A}}
\end{array}\right]^{T}}
\end{aligned}
$$

3.4 Modeling the system in a state space form with included PSS:

$$
\text { Same form in equation (12) but different states }
$$

$$
\begin{aligned}
& {[\Delta X i]=\left[\begin{array}{lllllll}
\Delta \hat{E}_{q} & \Delta \delta & \Delta \omega & \Delta E_{f d} & \Delta V_{W F} & \Delta V_{1} & \Delta V_{s}
\end{array}\right]^{T}} \\
& \left.\begin{array}{ccccccc}
\frac{-1}{K_{3} T_{d o}^{\prime}} & \frac{-K_{4}}{T_{d o}^{\prime}} & 0 & \frac{1}{T_{d o}^{\prime}} & 0 & 0 & 0 \\
0 & 0 & \omega_{s} & 0 & 0 & 0 & 0 \\
\frac{-K_{2}}{M} & \frac{-K_{1}}{M} & \frac{-K_{D} \omega_{s}}{M} & 0 & 0 & 0 & 0 \\
\frac{-K_{A} K_{6}}{T A} & \frac{-K_{A} K_{5}}{T A} & 0 & \frac{-1}{T_{A}} & 0 & 0 & \frac{K_{A}}{T A} \\
-\frac{K_{2} K_{P S S}}{M} & -\frac{K_{1} K_{P S S}}{M} & -\frac{K_{D} K_{P S S} \omega_{s}}{M} & 0 & -\frac{1}{T_{w}} & 0 & 0 \\
-\frac{K_{2} K_{P S S} T_{1}}{M T_{2}} & -\frac{K_{1} K_{P S S} T_{1}}{M T_{2}} & -\frac{K_{D} \omega_{s} K_{P S S} T_{1}}{M T_{2}} & 0 & \left(\frac{1}{T_{2}}-\frac{T_{1}}{T_{w} T_{2}}\right) & -\frac{1}{T_{2}} & 0 \\
-\frac{K_{P S S} K_{2} T_{1} T_{3}}{M T_{2} T_{4}} & -\frac{K_{P S S} K_{1} T_{1} T_{3}}{M T_{2} T_{4}} & -\frac{K_{P S S} K_{D} \omega_{s} T_{1} T_{3}}{M T_{2} T_{4}} & 0 & \left(\frac{T_{3}}{T_{4} T_{2}}-\frac{T_{3} T_{1}}{T_{4} T_{w} T_{2}}\right) & \left(\frac{1}{T_{4}}-\frac{T_{3}}{T_{2} T_{4}}\right) & -\frac{1}{T_{4}}
\end{array}\right]
\end{aligned}
$$

IV. Results and Discussions

From the block diagram of SMIB given in Fig. 1, PSS structure in fig. 3 and data obtained in appendix, the simulation is done in MATLAB /SIMULINK environment. Results obtained for variation in speed deviation. The variation in rotor speed is depicted in fig. 4, fig. 5, fig. 6 and fig. 7 for different operation points normal load, heavy load, light load and leading power factor. Simulation is run for $10 \mathrm{sec}$ and non adaptive algorithm is used for obtaining the characteristics with step function. From the fig (4) in case of normal load, it has been observed that the SMIB system without excitation and PSS is unstable. Also in fig. 5, fig. 6 and fig. 7 in cases of heavy and leading power factor the response of speed deviation going un stable and increase in 
magnitude without PSS, but after adding PSS damp out the oscillations during first cycle. Therefore from the simulation results, it is understood that the designed PSS is suitable for damping the oscillations by providing sufficient damping torque to the SMIB system.

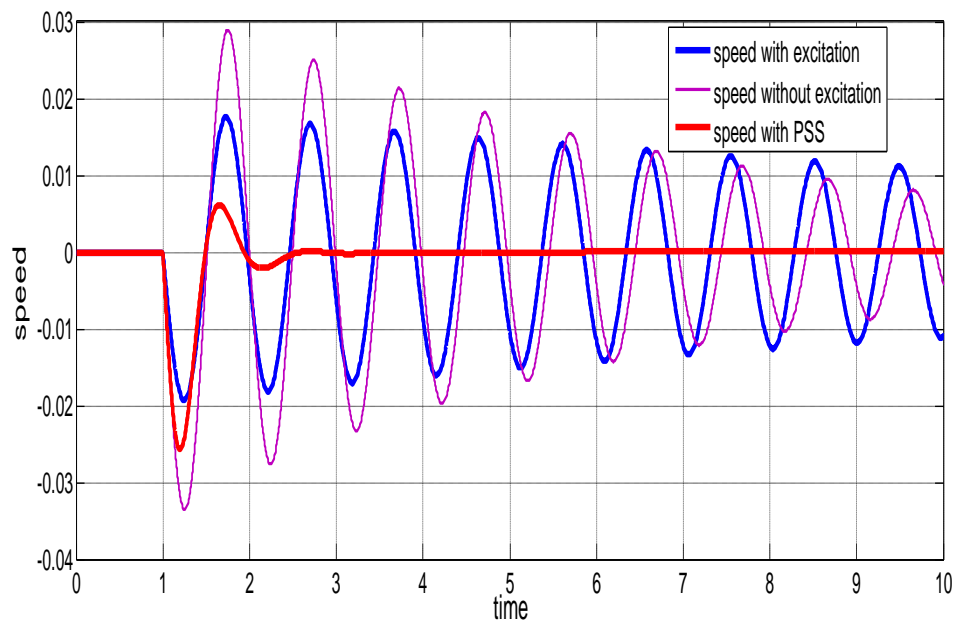

Figure 4 speed deviation response in case of normal load without PSS and with PSS

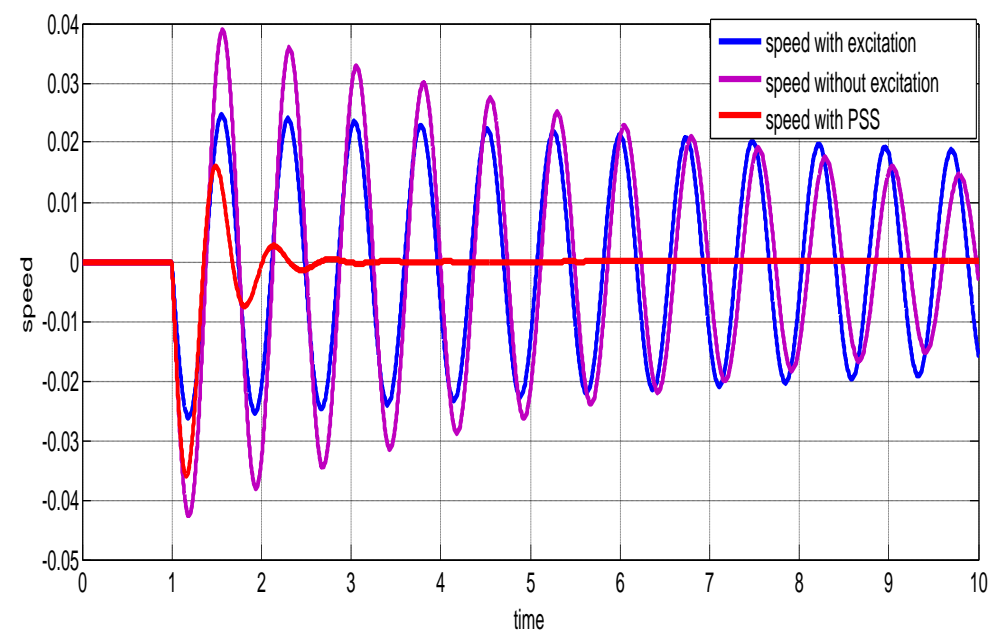

Figure 5 speed deviation response in case of heavy load without PSS and with PSS

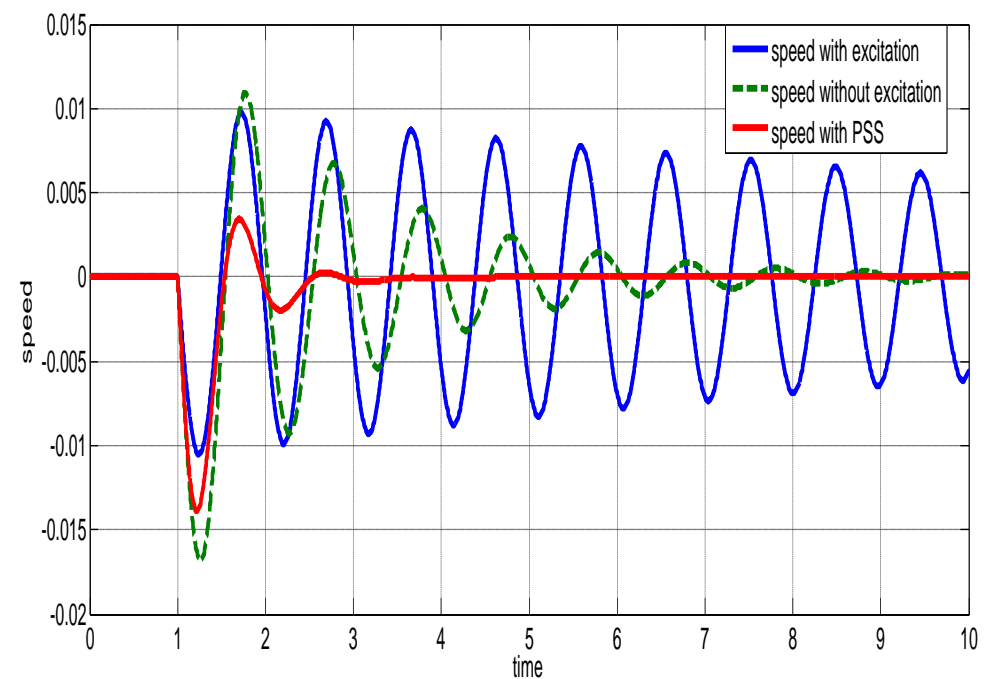

Figure 6 speed deviation response in case of light load without PSS and with PSS 


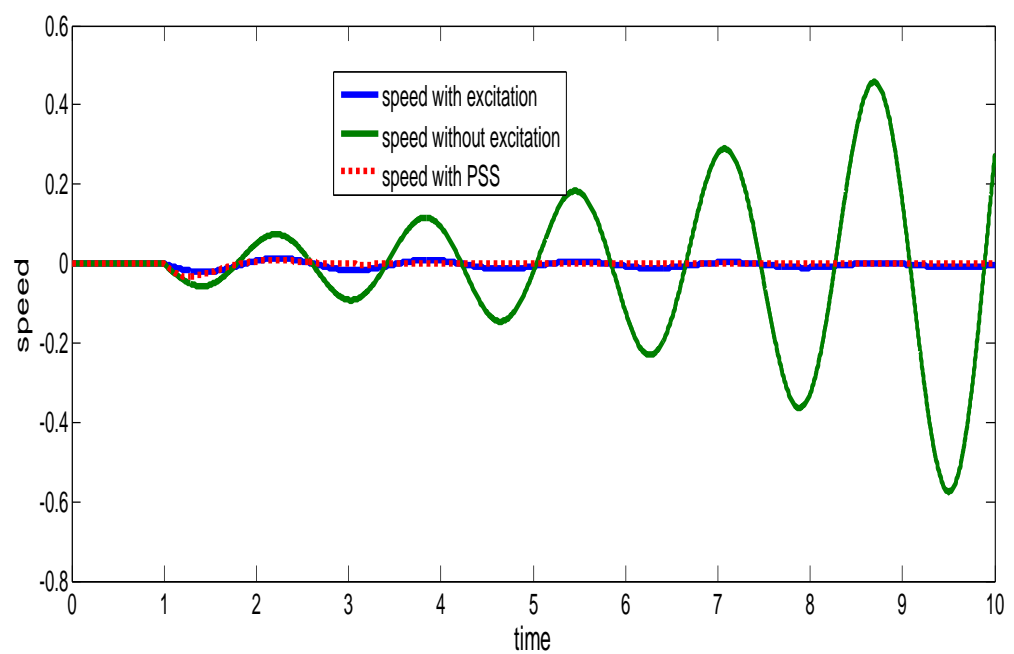

Figure 7 speed deviation response in case of leading power factor load without PSS and with PSS

\section{Conclusion}

This work proposes the design of PSS for single machine infinite bus (SMIB) system by eigenvalues technique to damp out oscillation in the test system described and it returned the system to the stable condition after the disturbance. The performance of PSS evaluated, by using time domain analysis and Eigenvalue analysis. Time domain analysis graphically shows the oscillations are damped out after the installation of PSS. It is found that designed controller provides good damping enhancement for various operating points of SMIB power system. The proposed PSS is very feasible, applied to multi machine power systems and easy to implement.

\section{References}

[1]. P. Kundur, Power system stability and control (New York, McGraw- Hill, 1994)

[2]. P. Kundur, W. Lei, Small signal stability analysis: Experiences, achievements, and challenges. International Conference on Power System Technology; 2002, 6-12.

[3]. F. P. DeMello and C. Concordia, Concepts of Synchronous Machine Stability as Affected by Excitation Control, IEEE Transactions on Power Apparatus and Systems, Vol. 88, 1969, 316-329.

[4]. P. Kundur, M. Klein, G. J. Rogers, and M. S. Zywno, Application of Power System Stabilizers for Enhancement of Overall System Stability, IEEE Transactions on Power Systems, Vol. 4, 1989, 614-626.

[5]. J. H. Chow, J. J. Sanchez-Gasca, Pole-placement Designs of Power System Stabilizers, IEEE Transactions on Power Systems, Vol. 4, No. 1, 271-277.

[6]. D. Mondal, A. Chakrabarti, A. Sengupta, Power System Small Signal Stability Analysis and Control (Academic Press 2014)

[7]. G. Rogers, Demystifying power system oscillations, IEEE Computer Applications in Power, vol. 9, no. 3, 1996,30 - 35. 